Case Report

\title{
Phagosyncope: an unusual presentation of airway malfunction due to acquired laryngomalacia in an elderly Indian male
}

\author{
Indrajit Suresh $^{1 *}$, Suhas Challa ${ }^{2}$, Sanjana Narendran Gattavadi ${ }^{3}$
}

\begin{abstract}
${ }^{1}$ Department of Pancreatology, ${ }^{2}$ Department of Internal Medicine, ${ }^{3}$ Department of Dentistry, CEG Hospital, Mysore, Karnataka, India
\end{abstract}

Received: 05 November 2020

Accepted: 10 December 2020

\author{
*Correspondence: \\ Dr. Indrajit Suresh, \\ E-mail: Indrajit.suresh@gmail.com
}

Copyright: $\odot$ the author(s), publisher and licensee Medip Academy. This is an open-access article distributed under the terms of the Creative Commons Attribution Non-Commercial License, which permits unrestricted non-commercial use, distribution, and reproduction in any medium, provided the original work is properly cited.

\begin{abstract}
Acquired laryngomalacia is an uncommon entity in adults. It may often be misdiagnosed, under-reported or confused with respiratory diseases such as bronchial asthma. The exact pathogenesis of acquired laryngomalacia remains poorly understood, although many at-risk groups have been identified. High pitched inspiratory stridor which worsens with physical exertion is often encountered in patients. Predominantly being a mechanical issue, wherein supraglottic structures occlude the airway, causing respiratory distress and hypoventilation, adult laryngomalacia may cause choking episodes. In the elderly, where mucosal laxity and poor neuromuscular control may be encountered, a possibility of this illness must be kept in mind in those presenting with respiratory symptoms. Supraglottoplasty remains the best mode of treatment currently and has a high success rate. The authors report a case of acquired laryngomalacia in an elderly male, who presented with an unusual cluster of complaints. He underwent detailed evaluation for confirmation of his diagnosis and underwent surgical correction, which ameliorated his condition.
\end{abstract}

Keywords: Laryngomalacia, Stridor, Phagosyncope, Dysphagia, Supraglottoplasty

\section{INTRODUCTION}

Syncope in the elderly is a significant problem associated with high morbidity and a two year mortality rate nearing 30 percent. $^{1}$ Approximately $5 \%$ of all acute medical admissions and 3\% of emergency hospital visits may be attributed to this problem. ${ }^{2}$

An increased understanding of human physiology, neuroautonomic mechanisms, neuropsychiatric and cardiovascular illnesses etc in the recent years, has allowed us to glimpse at the vast spectrum of syncope and its causes.

Mechanical causes of syncope are often of cardiovascular or respiratory origin, resulting in a demand-supply mismatch of oxygenated blood to the brain and culminating in a transient loss of consciousness. Previous researchers have described syncope during deglutition as relatively rare and typically being a neurally-mediated reflex.

Respiratory mechanical issues such as those encountered in laryngomalacia, cause inspiratory supraglottic collapse. ${ }^{3}$ A practical classification of laryngomalacia was suggested by Holinger et al in 1989 and remains highly relevant. ${ }^{4}$

Although laryngomalacia is the most common congenital lesion of the larynx, it may also be encountered in young adults and the elderly. ${ }^{5}$ In the elderly, the exact pathogenesis is still unknown but factors like relative supraglottic dilator muscle hypotonia, poor neuromuscular control and reflux disease have been postulated. An 
association with Parkinson's disease, amyotrophic lateral sclerosis, connective tissue disorders, trauma, radiotherapy, chronic inhaled steroid use, smoking or surgery may be present. ${ }^{6}$ In a few patients, no discernible cause may be found.

A high pitched inspiratory stridor which is position dependent and worsening with increased work of breathing is the most striking feature of this condition. Direct laryngoscopy or flexible nasopharyngolaryngoscopy may reveal an omega shaped epiglottis, short aryepiglottic folds and superfluous floppy mucosa over the arytenoid cartilages.

We report the case of an elderly gentleman who presented with syncopal episodes during eating, in whom the pathology was detected to be acquired type-1 laryngomalacia. The clinical features and management strategy adopted by us are described.

\section{CASE REPORT}

A 70 year old well nourished, overweight (BMI 29), south Indian gentleman presented to our hospital outpatient department with history of recurrent episodes of light headedness, choking sensation and nine episodes of transient loss of consciousness while consuming food. The episodes occurred over a two week period lasting between twelve to twenty seconds. Symptoms would occur sometimes within a few seconds of ingesting solid food, and there would be intermittent bouts of cough after consuming liquids. The patient reported better tolerance to soft diet. He also complained of exhaustion and disturbed sleep. He was taken to a local clinic on six occasions and was given salbutamol-ipratropium nebulisations and intravenous (IV) fluids. No episodes of hypoglycaemia or seizures were documented by the attending physicians. Blood pressure recordings were normal, while pulsoximetry showed oxygen saturation on three instances to be in the mid 80s- but improved with inhaled oxygen therapy. There was associated history of chronic gastroesophageal reflux disease, dyspnoea on exertion and noisy breathing during exertion and sleep. The patient was a known hypertensive and occasional smoker. His medications included cilnidipine and rabeprazole with domperidone. The patient was evaluated at a local clinic and a recommendation for BiPAP ventilation was given in view of probable chronic obstructive pulmonary disease (COPD) and obstructive sleep apnoea syndrome (OSAS). Electrocardiogram, echocardiogram, 24-hour holter monitoring, carotid artery Doppler, Electroencephalogram (EEG) and Magnetic resonance Imaging (MRI) of the brain were normal.

Chest X-ray as well as lateral cephalometry were normal. Pulmonary function test was performed and the ratio of FEV1 to peak expiratory flow (Empey index) was 12.6. A polysomnogram, conducted on an in-patient basis showed an apnoea-hypopnoea index (AHI) of 16 (Moderate).

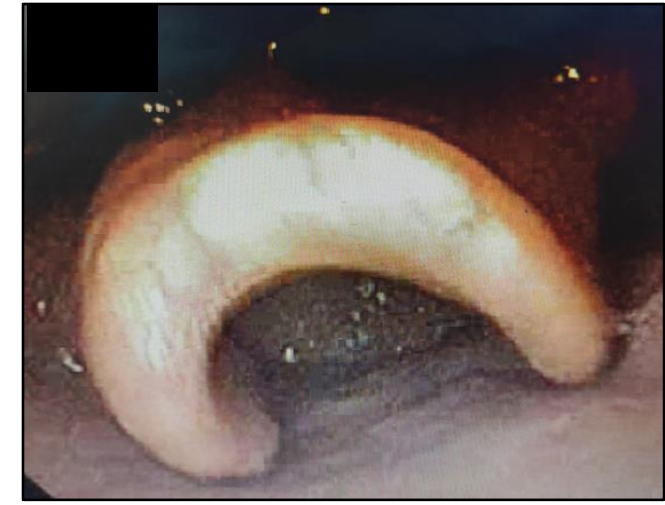

Figure 1: Omega shaped epiglottis.

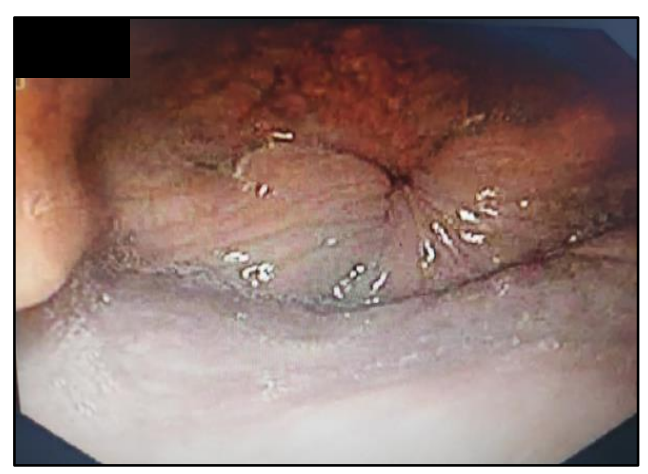

Figure 2: Occlusion of Rima glottidis by redundant aryepiglottic mucosa.

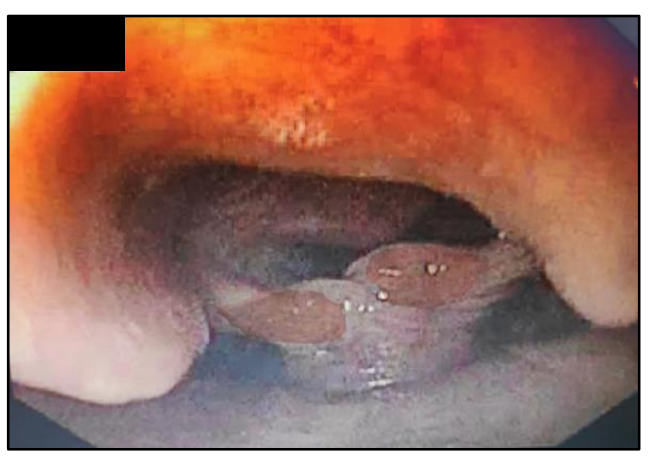

Figure 3: Post CO2 laser supraglottoplasty.

The patient was referred to our gastroenterology department where an upper GI endoscopy was done during which a non obstructing omega shaped epiglottis (Figure 1), prolapse of redundant mucosa overlying the aryepiglottic folds, corniculate and cuneiform cartilages into the laryngeal lumen causing near total obstruction of the rima glottidis during inspiration (Figure 2) along with evidence of gastroesophageal reflux disease and gastroduodenal erosions were observed.

Flexible laryngoscopy along with videostroboscopy confirmed the endoscopic findings and Vocal cord movements, ventricles as well as peri-laryngeal areas were found to be normal. Anterior rhinoscopy showed a mild deviation of the nasal septum to the right and a mildly 
enlarged inferior turbinate on the right. A provisional diagnosis of acquired laryngomalacia (ALM) type-1 was considered.

CO2 laser $(15600 \mathrm{~nm}, 3 \mathrm{~W}, 1 \mathrm{~Hz}, 400 \mathrm{~mm}$ focal length, $20 \times 0.5$ seconds exposure) supraglottoplasty was performed under general anesthesia by the surgeons of the otorhinolaryngology department. Redundant mucosa overlying the aryepiglottic folds was ablated sufficiently, and minimal bleeding was encountered (Figure 3).

Saline soaked surgical cottonoids were used to clean the charred mucosal remnants. Histopathological examination of the removed tissue showed congested and edematous squamous mucosa.

Following surgery, the patient was given intravenous antibiotics, proton pump inhibitor and corticosteroids. He complained of foreign body sensation in the throat necessitating performance of intermittent throat clearing manoeuvres. He was discharged on day 3 with oral corticosteroids, proton pump inhibitor, prokinetic and antibiotic for one week.

On follow-up after one month, the patient reported a significant amelioration of his complaints without any modes of assisted ventilation. No further episodes of phagosyncope were reported. Oral feeds of varying consistency were tolerated well. Sleep quality had also improved, and he did not feel exhausted during the day. A repeat pulmonary function test showed the Empey index at 9.8, and the polysomnogram AHI was 11 (mild). Since issues of gastroesophageal reflux persisted, he was prescribed oral baclofen $10 \mathrm{mg}$ twice daily for three weeks. During next follow-up after three weeks, reflux symptoms had also significantly reduced, and the AHI had improved to 7. Although, flexible laryngoscopy and videostroboscopy were recommended on follow-up, the patient refused to undergo the procedure.

\section{DISCUSSION}

Adult laryngomalacia- which is often acquired, is an uncommon diagnosis and the etiology, pathophysiology and clinical approach are poorly understood. Varying degrees of upper airway obstruction may be encountered in adults and may be caused by a long posteriorly prolapsing curled epiglottis and the presence of short bulky arytenoids with superfluous, hyperactive, hypotense or floppy supraglottic tissue. ${ }^{7}$ These features may be seen in combination or separately. ALM is also a rare cause of Obstructive sleep apnoea syndrome (OSAS). ${ }^{8}$

Respiratory compromise worsens in individuals with ALM, during exertional activities, stages 3 and 4 of delta sleep etc. During these phases, there may be associated stridor, hypopnoea, intermittent hypoxia and hypercapnia. ${ }^{9}$

Robust theories implicating structural and neuromuscular aetiologies for ALM have been proposed, and in many cases there may be significant overlap amongst the two especially in adults. Poorly coordinated respiratory drive and impaired airflow dynamics with errant mucosal mobility and increased airway resistance may just represent one half of the problem while predisposing factors such as mucosal laxity associated with advanced age, mucosal edema secondary to chronic smoking, post nasal drip and GERD represent the other. ${ }^{10}$

In our patient, chronic gastro-esophageal reflux, advanced age and smoking were likely contributory to ALM. A detailed workup schema for the elderly patient who presents with phagosyncope must rule out other anomalies in the cardiovascular and neurologic systems as well. Possibility of upper airway compromise should always be kept as a differential diagnosis in patients with clinically significant dyspnea, stridor or sleep apnea. The Empey index is a bedside tool that can help attending physicians in predicting upper airway compromise and is a simple calculation of the ratio of forced expiratory volume (FEV1 in $\mathrm{ml}$ ) to the Peak expiratory flow rate (PEFR in litres per min). Patients with upper airway obstruction tend to have values greater than 10 and higher ratios correlate well with severe obstruction. ${ }^{11}$

The initial provisional diagnosis of COPD with OSAS was erroneous and the suggested BiPAP ventilation strategy would not have sufficed for managing the patient's illness and his syncopal episodes would have likely continued with catastrophic consequences.

Laryngoscopy is currently the most accurate test in ALM and can demonstrate supraglottic collapse that may be the result of indrawing of superfluous mucosa overlying the arytenoid cartilages, aryepiglottic folds or ventricular folds during deep inspiration. It can also help the surgeon determine the approach which would benefit the patient.

Over the last few decades, advances in surgical otorhinolaryngology have enabled the use of lasers and radiofrequency ablation (RFA) over traditional cold steel techniques. Supraglottoplasty is the preferred procedure in ALM, and was first described by Lane et al in $1984 .{ }^{12} \mathrm{It}$ has a success rate of $70 \%$ to $100 \% .{ }^{13}$ The type of lesion determines the approach in supraglottoplasty which may be epiglottopexy, epiglottoplasty, aryepiglottoplasty or arytenoidoplasty. ${ }^{14}$ In our patient, a CO2 laser ablation technique was used remove excessive mucosa over the aryepiglottic folds, and was associated with minimal bleeding. The decision to avoid any unnecessary surgery involving the epiglottis was made after much deliberation among the surgeons and pulmonologist. As the epiglottis was not patulous nor prolapsing into the airway, aryepiglottoplasty was deemed sufficient. The preference of techniques like laser, coblation or harmonic shears depends upon the individual surgeon. Post-operative care must be taken to ameliorate mucosal edema and stridor. Inhaled steroids, adrenaline, anti-reflux therapy and oxygen administration may be required post-operatively for a short while. History of symptomatic improvement, 
reduction in the Empey index as well as improvement in the AHI provided reliably good information on the effectiveness of the procedure, and may be used as long term quality of life indicators. A follow up flexible laryngoscopy alongwith videostroboscopy is a preferred suggestion to be made to the patients after a month or at any time if hoarseness develops.

\section{CONCLUSION}

ALM, although encountered uncommonly should be considered as a differential diagnosis in adults presenting with features of airway malfunction. Misdiagnosis as bronchial asthma is commonly encountered. The causes of ALM may be multifactorial, and detailed workup including laryngoscopy/videostroboscopy should be done. Supraglottoplasty is the procedure of choice for ALM and may be performed by various methods with good success rate and improvement in the patient's quality of life.

Funding: No funding sources Conflict of interest: None declared

Ethical approval: Not required

\section{REFERENCES}

1. Patel PR, Quinn JV. Syncope: a review of emergency department management and disposition. Clin Exp Emerg Med. 2015;2(2):67-74.

2. Kapoor WN. Evaluation and management of the patient with syncope. JAMA. 1992;268(18):2553-60.

3. Holinger LD, Volk MS, Tucker GF. Congenital laryngeal anomalies associated with tracheal agenesis. Ann Otol Rhinol Laryngol. 1987;96(5):505-8.

4. Holinger LD, Konior RJ. Surgical management of severe laryngomalacia. Laryngoscope. 1989;99(2):136-42.

5. Siou GS, Jeannon JP, Stafford FW. Acquired idiopathic laryngomalacia treated by laser aryepiglottoplasty. J Laryngol Otol. 2002;116(9):733-35.
6. Dion GR, Eller RL, Thomas RF. Diagnosing aerodynamic supraglottic collapse with rest and exercise flexible laryngoscopy. Journal of Voice. 2012;26(6):779-84.

7. Woo P. Acquired laryngomalacia: epiglottis prolapse as a cause of airway obstruction. Ann Otol Rhinol Laryngol. 1992;101(4):314-20.

8. Rodriguez Adrados F, Esteban Ortega F, Peña Griñán N. Massive hyperplasia of the arytenoid mucosa with sleep apnea and stridor. Endoscopic resection by $\mathrm{CO} 2$ laser. Acta Otorrinolaringol Esp. 1999;50(8):664-66.

9. Rutt AL, Dworkin JP, Stern N. Supraglottic Obstruction in an Adult with Inspiratory Arytenoid Cartilage Prolapse. Otolaryngology. 2014;4:165.

10. Belmont JR, Grundfast K. Congenital laryngeal stridor (Laryngomalacia): etiologic factors and associated disorders. Ann Otol Rhinol Laryngol. 1984;93(5 Pt 1):430-37.

11. Beaty MM, Wilson JS, Smith RJ. Laryngeal motion during exercise. Laryngoscope. 1999;109(1):136-39.

12. Lane RW, Weider DJ, Steinem C. Laryngomalacia. A review and case report of surgical treatment with resolution of pectus excavatum. Arch Otolaryngol. 1984;110:546-51.

13. Escher A, Probst R, Gysin C. Management of laryngomalacia in children with congenital syndrome: the role of supraglottoplasty. J Pediatr Surg. 2015;50:519-23.

14. Craig S, Navarrete E. Laser supraglottoplasty for laryngomalacia: are specific anatomical defects more influential than associated anomalies on outcome?. Inter J of Ped Oto. 2001;57:235-244.

Cite this article as: Suresh I, Challa S, Gattavadi SN. Phagosyncope: an unusual presentation of airway malfunction due to acquired laryngomalacia in an elderly Indian male. Int J Otorhinolaryngol Head Neck Surg 2021;7:170-3. 